Linping Wang, Kengo Shigetomi*, Keiichi Koda, Aori Gele and Yasumitsu Uraki

\title{
A branched structure provides kraft lignins a denser morphology and a high molar mass for a given hydrodynamic radius
}

https://doi.org/10.1515/hf-2019-0292

Received November 21, 2019; accepted February 14, 2020

\begin{abstract}
Size exclusion chromatography with multiangle laser light-scattering detectors was applied for acetylated softwood and hardwood kraft lignins (Ac-SKL and Ac-HKL) and 8-O-4' type of linear polymeric lignin model (Ac-M-804') to compare their swelling behaviors. The plot of molar mass (MM) vs. retention time for Ac-M-804' was similar to that of polystyrene, which revealed that Ac-M$804^{\prime}$ exhibited swelling behavior that was similar to that of polystyrene. However, the MM values of both Ac-KLs were larger than those of polystyrene standards at any retention time. This difference indicated that both Ac-KLs had a more compact structure than those of polystyrene and Ac-M-804'. One hypothesis is that the larger MM of both Ac-KLs stems from their branched structures. To verify this hypothesis, the frequency of $5-5$ ' interunit linkage in lignin samples was determined by ${ }^{1} \mathrm{H}$ NMR after nitrobenzene oxidation. A linear relationship between MM and 5-5' abundance was observed in the high MM region.
\end{abstract}

Keywords: absolute molar mass, biomass, branching frequency, kraft lignin, structure elucidation

\section{Introduction}

Lignin is an aromatic polymer that is composed of phenylpropane units; it is the second most abundant biomass component after cellulose in terrestrial plants.

\footnotetext{
*Corresponding author: Kengo Shigetomi, Research Faculty of Agriculture, Hokkaido University, Sapporo 060-8589, Japan, e-mail: sgtm@for.agr.hokudai.ac.jp. https://orcid.org/0000-00019560-8091

Linping Wang: Graduate School of Agriculture, Hokkaido University, Sapporo 060-8589, Japan

Keiichi Koda and Yasumitsu Uraki: Research Faculty of Agriculture, Hokkaido University, Sapporo 060-8589, Japan

Aori Gele: State Key Laboratory of Pulp and Paper Engineering, South China University of Technology, Guangzhou, Guangdong 510640, China
}

Approximately 50 million tons of technical lignins are annually produced from the paper industry, and most of them are burned for energy. Although extensive efforts have been devoted to their value-added utilization, structural complexity is an obstacle for development. Lignin has a highly variable structure in terms of its phenylpropane units, interunit linkages and molar mass (MM), which depend on the wood species, seasonality and geographical location as well as the delignification process. With the recent development of the nuclear magnetic resonance (NMR) technique, the monomer compositions, functional groups and interunit linkages of different types of lignins have been well elucidated (Capanema et al. 2001; Ralph et al. 2004; Crestini et al. 2011; Santos et al. 2011). However, accurate MM determination is still arguable despite its importance in the prediction of the mechanical and rheological properties.

Size-exclusion chromatography (SEC) with the calibration curve of authentic standards (e.g. polystyrene) has been widely used to determine the relative MM of both intact (Lindströmn 1979; Gosselink et al. 2004; Saito et al. 2014) and acetylated (Siochi et al. 1990; Glasser et al. 1993; Baumberger et al. 2007; Alekhina et al. 2015; Lourençon et al. 2015) lignins. However, the method has an inherent limitation. Specifically, when the swelling behavior or hydrodynamic radius of the sample is very similar to that of standard polymer, the value is reliable.

SEC combined with multi-angle laser light-scattering detectors (SEC-MALS) is an attractive method to determine the absolute MM independently of the polymer swelling behavior. Recently, an SEC-MALS system with a laser light of a longer wavelength (785 nm) and bandpass filters has been developed (Zinovyev et al. 2018). This system appears to be suitable for the lignin MM determination because the autofluorescence of lignin can be considerably eliminated. Zinovyev et al. reported MMs of different types of intact isolated lignins in a dimethyl sulfoxide (DMSO) /LiBr solvent using the above-mentioned apparatus, and the obtained MMs of technical lignins were higher than those from the conventional SEC with a calibration curve of authentic polystyrene sulfonates (Zinovyev et al. 2018). Our research group used this apparatus to obtain the Mw and Mn values of Ac-HKL in tetrahydrofuran (THF) 
because acetylated lignin preparations in THF were widely investigated in the conventional SEC system without a light-scattering detector. In this experiment, the MM of Ac-HKL obtained by the SEC-MALS system was much larger than that obtained by a conventional SEC system with a calibration curve of polystyrene (Wang et al. 2019). These differences in MM were attributed to the different swelling behaviors of lignin preparations and authentic standard samples. Intrinsic viscosity $([\eta])$ is related to the gyration radius of polymer in the solution. According to the Mark-Houwink-Sakurada equation, $[\eta]$ is expressed as $[\eta]=K M^{a}$. Therefore, exponent " $a$ " significantly influences the gyration radius. Thus far, the " $a$ " values of several lignins in different solvents have been shown to be in the range of 0.12-0.32 (Goring 1971). These low " $a$ " values were caused by the compact solution structure of lignin molecules. The " $a$ " value of Ac-HKL in THF was 0.24 according to the previous study (Wang et al. 2019). Because the " $a$ " value of polystyrene is 0.725 in THF (Alliet and Pacco 1968; Spatorico and Coulter 1973), Ac-HKL has a more compact structure than that of polystyrene. Therefore, the conventional SEC with a calibration curve of polystyrene standards provides incorrect MM.

According to the study by Lyulin et al. (2001), the polymer with higher branching points has a more compact structure. Lue reported that dendritic polymer has a more compact structure compared to linear polymer with the same MM (Lue 2000). One hypothesis is that the compact solution structure of kraft lignin (KL) may result from its branched structure. To verify this hypothesis, in this study, the SEC-MALS analysis of acetylated 8-O-4'-type linear polymeric lignin model (Ac-M-804') in addition to softwood kraft lignins (SKLs) and hardwood kraft lignins (HKLs) was conducted to compare the swelling behavior of practical lignins with that of the linear lignin model. This lignin model was composed of only an 8-0-4' interunit linkage, a predominant linkage in the native lignin, and hence this can be the most suitable material as a linear polymer reference. Among the interunit linkage of lignin, $5-5^{\prime}$ and $4-O-5^{\prime}$ are proposed to be the branching points. Although the frequency varies based on wood species and determination methods, the 5-5' frequency (19-27\%) of softwood lignin determined by permanganate oxidation, ${ }^{13} \mathrm{C}$ NMR and UV spectroscopy (Pew 1963; Bose et al. 1998; Capanema et al. 2004) was still much larger than the 4-O-5' frequency $(4-5 \%)$ determined by $\mathrm{CuO} / \mathrm{NaOH}$ permanganate oxidation (Erickson et al. 1973) and thioacidolysis/31P NMR (Smit et al. 1997), which is well reviewed and reported by Chang and Jiang (2020). In addition to these methods, the alkaline nitrobenzene oxidation method combined with ${ }^{1} \mathrm{H}$ NMR spectroscopy or gas chromatography is possible to detect the 5-5' structure from wood sawdust (Katahira and Nakatsubo 2001; Tamai et al. 2015). In this study, using KLs as a branched polymer, the frequency of the 5-5' linkage in the lignin preparations was determined by a combinational method of alkaline nitrobenzene oxidation and ${ }^{1} \mathrm{H}$ NMR.

\section{Materials and methods}

Sample preparation for the SEC-MALS analysis: HKL and SKL were precipitated by acidification from black liquor of Eucalyptus exserta F.Muell (supplied by Hainan Jinhai Pulp Paper Co., Ltd., Danzhou, China) and industrial black liquor of several Hokkaido coniferous woods (supplied by Oji Paper Co., Ltd., Tomakomai, Japan), respectively. The precipitates were collected by filtration and washed with distilled water until $\mathrm{pH}$ 3. The resultant precipitates were dried in air, and then in vacuo at $50^{\circ} \mathrm{C}$ for $48 \mathrm{~h}$ to obtain HKL and SKL powders. The 8-O-4'-type polymeric lignin model (M-804') was prepared according to previous studies (Kishimoto et al. 2006, 2008a,b). The procedure is shown in a simple manner in Scheme 1. The HKL, SKL and M-804' powders were acetylated with acetic anhydride in pyridine at room temperature for $48 \mathrm{~h}$. The acetylated samples were precipitated by pouring the mixture into distilled water with ice, and the precipitates were collected by centrifugation. The precipitates were further washed with distilled water followed by lyophilization to yield Ac-HKL, Ac-SKL and Ac-M-804'.

MALDI-TOF MS measurement: Matrix-assisted laser desorption/ ionization-time-of-flight mass spectrometry (MALDI-TOF MS) measurement was performed on an Applied Biosystems Voyager DE-STR instrument (Foster City, CA, USA) operating in the positive-ion linear mode with a pulsed UV laser beam (nitrogen laser, $A=337 \mathrm{~nm}$ ). The accelerating voltage was $20 \mathrm{kV}$. M-804' was first dissolved in acetonewater $(9: 1, \mathrm{v} / \mathrm{v})$ at $0.1 \mathrm{mg} \mathrm{ml}^{-1}$. A total of $1.5 \mu \mathrm{l}$ of the matrix solution containing $20 \mathrm{mg} \mathrm{ml}^{-1}$ of 2,5-dihydroxybenzoic acid and $4.2 \mathrm{mg} \mathrm{ml}^{-1}$ of $\mathrm{LiCl}$ in Milli-Q water (Millipore, Temecula, CA, USA) was mixed with the same amount of sample solution on a target plate. The measurement was performed after the removal of solvent by air.

Determination of MM by SEC-MALS: SEC-MALS measurements were carried out on a high-performance liquid chromatography (HPLC) system (Shimadzu LC-10, Kyoto, Japan) equipped with a MALS detector (DAWN HELEOS 8, Wyatt Technology, Santa Barbara, CA, USA) and a differential refractive detector (Shimadzu RID-10, Kyoto, Japan). The laser light wavelength of MALS was $785 \mathrm{~nm}$, and bandpass filters were placed in front of all eight detectors. The temperature of the column oven was set to $35^{\circ} \mathrm{C}$. THF was used as an eluent at a flow rate of $0.5 \mathrm{ml} \mathrm{min}^{-1}$. A sample injection volume was $100 \mu \mathrm{l}$. The column used was Shodex GPC KF-804L (Showa Denko Co. Ltd., Tokyo, Japan), and two columns were connected in series. A polystyrene calibration curve was created by plotting the MM of 18 authentic polystyrene standards with MM values in the range of 580-4 $226000 \mathrm{~g} \mathrm{~mol}^{-1}$ against their retention time. Hydrodynamic radius, " $\mathrm{r}$ ", at each retention time was calculated based on the universal 


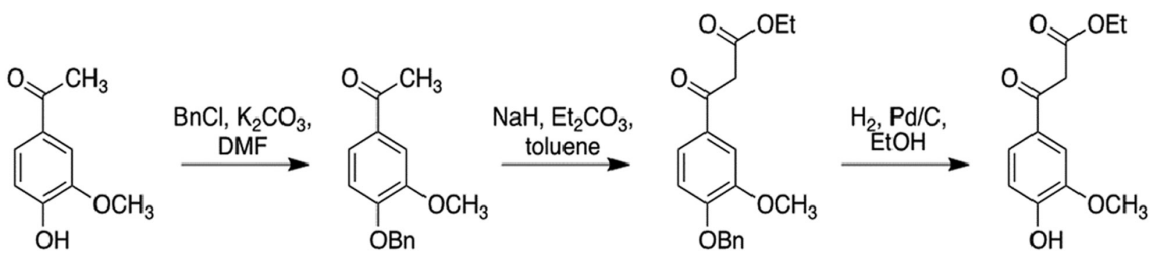

Acetovanillone
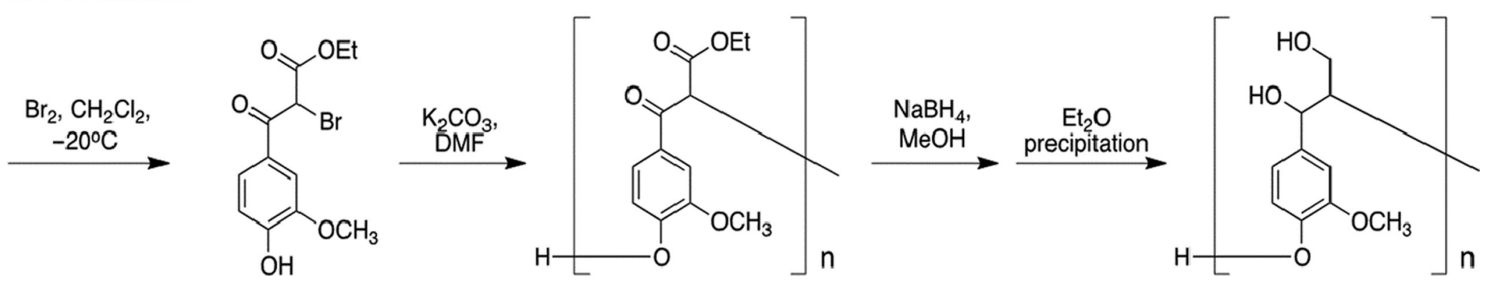

Scheme 1: Synthesis of the 8-0-4'-type polymeric lignin model.

calibration curve " $\log [\eta] M$ vs. retention time" of polystyrene standards and a derived formula, $[\eta] M=2.5 N_{\AA} V_{e}$, deduced from Einstein's equation, $\eta=\eta_{0}\left(1+2.5 v_{2}\right)$, where $N_{\mathrm{A}}$ is Avogadro's number, $V_{e}$ is the equivalent volume of the spherical molecule, $\eta$ is the dynamic viscosity of the solution, $\eta_{0}$ is the viscosity of the solvent, and $v_{2}$ is the volume fraction of spheres. The specific refractive index increments $(d n / d c)$ of all lignin samples for MM calculation were determined on an automatic refractometer (Abbemat 550, Anton Paar, Graz, Austria). The wavelength of the polarized light was $589 \mathrm{~nm}$. Samples with different concentrations in THF were loaded into a sample cell at $25^{\circ} \mathrm{C}$. The $d n / d c$ values, which were calculated from the tangent for the plot of the refractive index against concentration, were $0.175 \mathrm{ml}$ $\mathrm{g}^{-1}$ for Ac-HKL, $0.167 \mathrm{ml} \mathrm{g}^{-1}$ for Ac-SKL and $0.127 \mathrm{ml} \mathrm{g}^{-1}$ for Ac-M-8O4'.

Quantification of the 5-5' $^{\prime}$ interunit linkage of lignins and their fractions: KL fractions were prepared according to previous studies (Cui et al. 2014; Wang et al. 2019). Briefly, KLs were separated into acetone insoluble (AI-SKL and AI-HKL) and acetone soluble (AS-SKL and AS-HKL) parts, and AS-KLs were subsequently fractionated with acetone containing 20\% hexane (AS-SKL20 and AS-HKL20), followed by $40 \%$ hexane (AS-SKL40 and AS-HKL40). A total of $25 \mathrm{mg}$ of each sample was reacted with $4 \mathrm{ml}$ of a $2 \mathrm{M}$ $\mathrm{NaOH}$ solution and $0.24 \mathrm{ml}$ of nitrobenzene in a $20-\mathrm{ml}$ stainless steel vessel for $2 \mathrm{~h}$ at $170^{\circ} \mathrm{C}$ in an oil bath. The reaction mixture was cooled to room temperature by immersion in running water. Then, $0.5 \mathrm{ml}$ of 1,4-dioxane containing $2.5 \mathrm{mg}$ of 5-iodovanillin as an internal standard was added to the reaction mixture. The mixture was filtered, and the residue was washed with $0.2 \mathrm{M} \mathrm{NaOH}(1 \mathrm{ml} \times 3)$. The filtrate and washings were combined and acidified to $\mathrm{pH} 2-3$ with a $0.5 \mathrm{~N} \mathrm{HCl}$ solution. Then, the solution was extracted with ethyl acetate $(30 \mathrm{ml} \times 3)$, and the organic layer was washed with brine and dried over $\mathrm{Na}_{2} \mathrm{SO}_{4}$. The products were acetylated with $2 \mathrm{ml}$ of acetic anhydride/pyridine $(1: 1, \mathrm{v} / \mathrm{v})$ at $50^{\circ} \mathrm{C}$ for $2 \mathrm{~h}$. After removing the acetylating reagents by evaporation with toluene, the acetylated products were dried in vacuum at room temperature. The resultant sample was dissolved in chloroform- $d$ with tetramethylsilane as an internal standard and analyzed by ${ }^{1} \mathrm{H}$ NMR $(270 \mathrm{MHz})$ on JNM-EX270 (JEOL, Tokyo, Japan). The products were quantitatively analyzed based on the aldehyde peak areas appearing at $9.86 \mathrm{ppm}, 9.90 \mathrm{ppm}$, $9.94 \mathrm{ppm}$ and $9.95 \mathrm{ppm}$ by deconvolution. The detailed process is shown in Scheme 2.

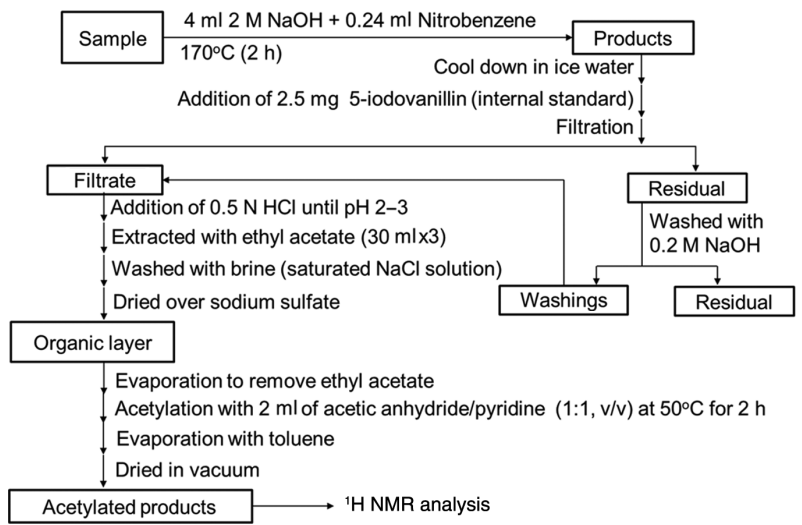

Scheme 2: Determination of the 5-5' interunit linkage by nitrobenzene oxidation and ${ }^{1} \mathrm{H}$ NMR.

\section{Results and discussion}

\section{SEC-MALS measurement of three acetylated lignin samples}

Figure 1a shows the structure of a synthetic 8-0-4'-type linear polymeric lignin model (M-804') and its MALDITOF mass spectrum. The molecular ion peaks were observed at intervals of $\mathrm{m} / z 196$, and the value represents well the mass of the repeat unit. However, the signal intensities were decreased with an increase in the degree of polymerization (DP), and hardly any signal was observed at DP $>20$. Figures $1 b, c$ and d show the SEC-MALS- refractive index (RI) profiles of acetylated M-804' and KLs. In each figure, the left $\mathrm{Y}$ axis shows the detector voltages of MALS and RI responses, and the right $\mathrm{Y}$ axis shows the MM. Line 1 is the polystyrene calibration curve, and line 2 is the MM curve of the test 

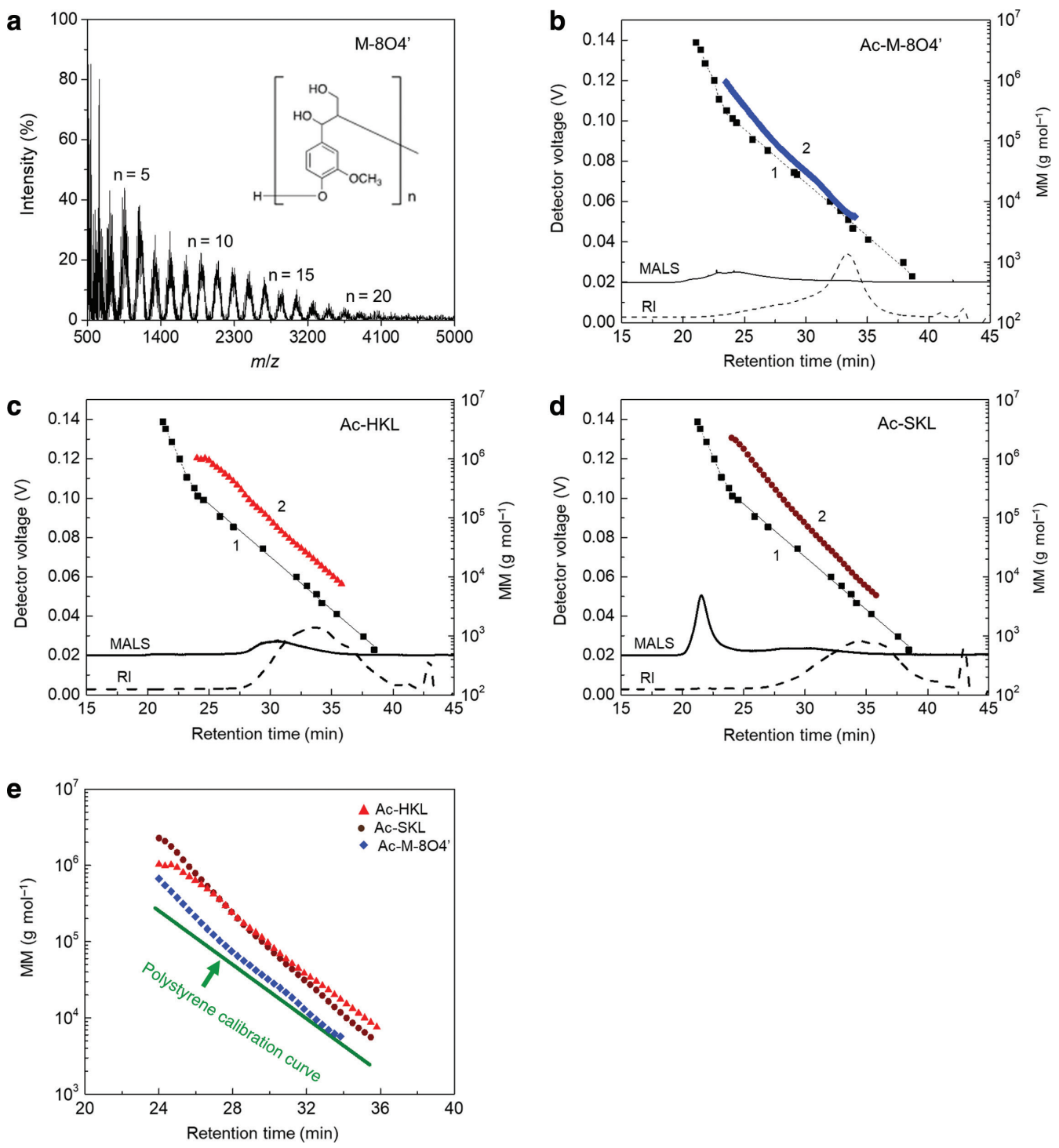

Figure 1: Structure of MALDITOF mass spectrum and SEC-MALS- refractive index (RI) profiles of acetylated M-804' and KLs.

(a) MALDI-TOF mass spectrum of M-804', SEC-MALS-RI chromatograms of (b) Ac-M-804', (c) Ac-HKL, (d) Ac-SKL, and (e) overlaid MM curves of three lignin samples. In (b-d), the left $Y$ axis represents the detector voltage of RI and MALS responses. The right $Y$ axis represents MM. Solid lines show chromatograms that are monitored by MALS, where the detector is located at $90^{\circ}$ to the laser light. Dashed lines show the chromatograms monitored by RI. Line 1 shows the calibration curves of polystyrene standards. Line 2 shows the absolute MM obtained by MALS with band filters.

samples. In three subfigures, the MALS signals always appeared at an earlier retention time than the RI signals, which indicates the higher sensitivity of the light-scattering detector for larger MM molecules. In Figure 1b, Ac-M-804' exhibited the peak top MM (Mp) at $6.4 \times 10^{3}$ $\mathrm{g} \mathrm{mol}^{-1}$ in RI, and the RI response was observed even at the higher MM region $\left(10^{4}-10^{6} \mathrm{~g} \mathrm{~mol}^{-1}\right)$. This result is not consistent with that of MALDI-TOF-MS. Although MALDITOF-MS is a powerful technique especially for qualitative analyses, the method does not completely cover the actual MM distribution or the highest MM molecule within the polymer sample. Thus, the conventional SEC system still remains irreplaceable for the MM measurement of the polymers.

The MM curves of Ac-M-804' and acetylated KLs were obtained by MALS responses and RI increment $(d n / d c)$ values independently measured (see the Experimental section of "Determination of MM by SEC-MALS"). As shown in Figure 1b, the MM curve of Ac-M-804' was almost similar to the calibration curve prepared with polystyrene standards. This result shows that Ac-M-804' has a swelling behavior similar to that of polystyrene in THF, and the predominant 8-O-4' substructure in lignins does not contribute to the discrepancy in MM. In contrast, the MM values 
of Ac-SKL and Ac-HKL were approximately 5- to 10-fold larger than the MM value of polystyrene at any retention time (Figure $1 \mathrm{c}$ and $\mathrm{d}$ ), which produces their distinctly different swelling behaviors (i.e. the more compact structure of Ac-KLs than that of linear polymers). This tendency was also observed between underivatized lignins and polystyrene sulfonates in DMSO/LiBr (Zinovyev et al. 2018). Although the results from different solvent systems are not directly comparable, it can be speculated that the compact morphology of KLs does not stem from free hydroxyl functionalities that may form intramolecular hydrogen bonds. To better compare the MM of these three lignin samples, MM vs. retention time curves were overlaid in Figure 1e. Figure 1e illustrates an analogy of swelling behavior of Ac-SKL and Ac-HKL as well as their difference with linear compounds. The result clearly indicates that the swelling behavior of acetylated lignins is independent of guaiacyl (G)/syringyl (S) monomeric compositions. Overall, these observations implied the involvement of other structural features in compact morphologies of KLs and strongly motivated us to examine the branched substructure effect.

\section{Determination of the 5-5' abundance of lignin and their fractions}

Katahira and Nakatsubo reported the application of alkaline nitrobenzene oxidation combined with ${ }^{1} \mathrm{H}$ NMR spectroscopy for the detection of the 5-5' substructure in sawdust samples (2001). Although the yields of nitrobenzene oxidation products from KLs are known to be lower than those from native samples (11-14\% and 20-26\%, respectively) (Villar et al. 1997; Tarabanko and Tarabanko 2017), this method was applied as a facile strategy to estimate $5-5^{\prime}$ abundance, with limiting the comparison only to the KL preparations. The formyl proton signals of acetylated nitrobenzene oxidation products from M-804', SKL and HKL are shown in Figure 2. The signals corresponding to the 5-5' type, G-type and S-type products appear at $9.95 \mathrm{ppm}, 9.94 \mathrm{ppm}$ and $9.90 \mathrm{ppm}$, respectively. Because the signals of 5-5' and G-types partly overlap, the peaks were deconvoluted prior to quantification. The molar concentrations ( $\mathrm{MC}, \mathrm{mmol} \mathrm{g}^{-1}$ ) of these products were calculated based on the internal standard (i.e. acetylated 5-iodovanillin), according to their integrated peak areas. The abundance of 5-5' interunit linkage in respective nitrobenzene oxidation products were calculated based on Eq.1

$$
5-5^{\prime} \text { abundance }=\mathrm{MC}_{5-5^{\prime}} /\left(2 \mathrm{MC}_{5-5^{\prime}}+\mathrm{MC}_{\mathrm{G}}+\mathrm{MC}_{\mathrm{S}}\right) \times 100
$$

To obtain a more detailed information on the branching frequency of lignin, the fractionation of two KLs was performed by solvent precipitation using acetone and hexane following the procedure reported by Cui et al. (Cui et al. 2014). The RI chromatogram of each acetylated

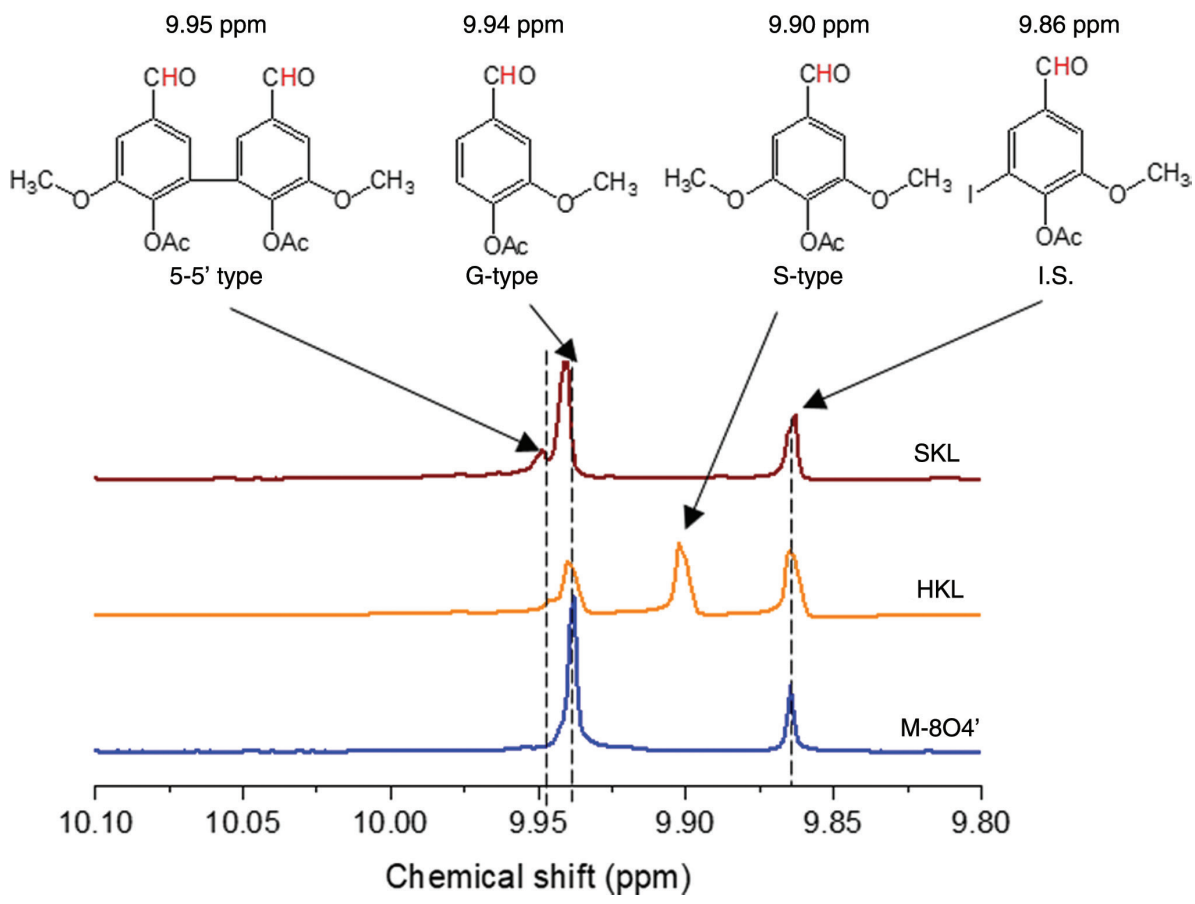

Figure 2: ${ }^{1} \mathrm{H}$ NMR analysis of acetylated nitrobenzene oxidation products. 
fraction is shown in Figure 3. In both SKL and HKL, the precipitation successfully provided the fractions with a different MM distribution and different Mp.

The 5-5' abundances of these fractions were also estimated by the ${ }^{1} \mathrm{H}$ NMR analysis of alkaline nitrobenzene oxidation products. Table 1 shows the detailed molar concentration and 5-5' abundance of KLs and their fractions. The 5-5' abundance of SKL is 17.4 per $100 \mathrm{Ar}$, which is comparable to the reported values of other softwood lignin (20-28 per 100 Ar) (Chang and Jiang 2020). The lower value of 5-5' abundance in the present study might stem from the low quantity of nitrobenzene oxidation of SKL as the benzylic oxygen function of SKL is less abundant than that of native lignin. In all fractions, SKLs showed a higher 5-5' abundance than those of HKLs. This result reflects well the predominance of the guaiacyl unit in original coniferous lignin, and the structure of SKL should be more branched than that of HKL. When focusing on different fractions, the 5-5'
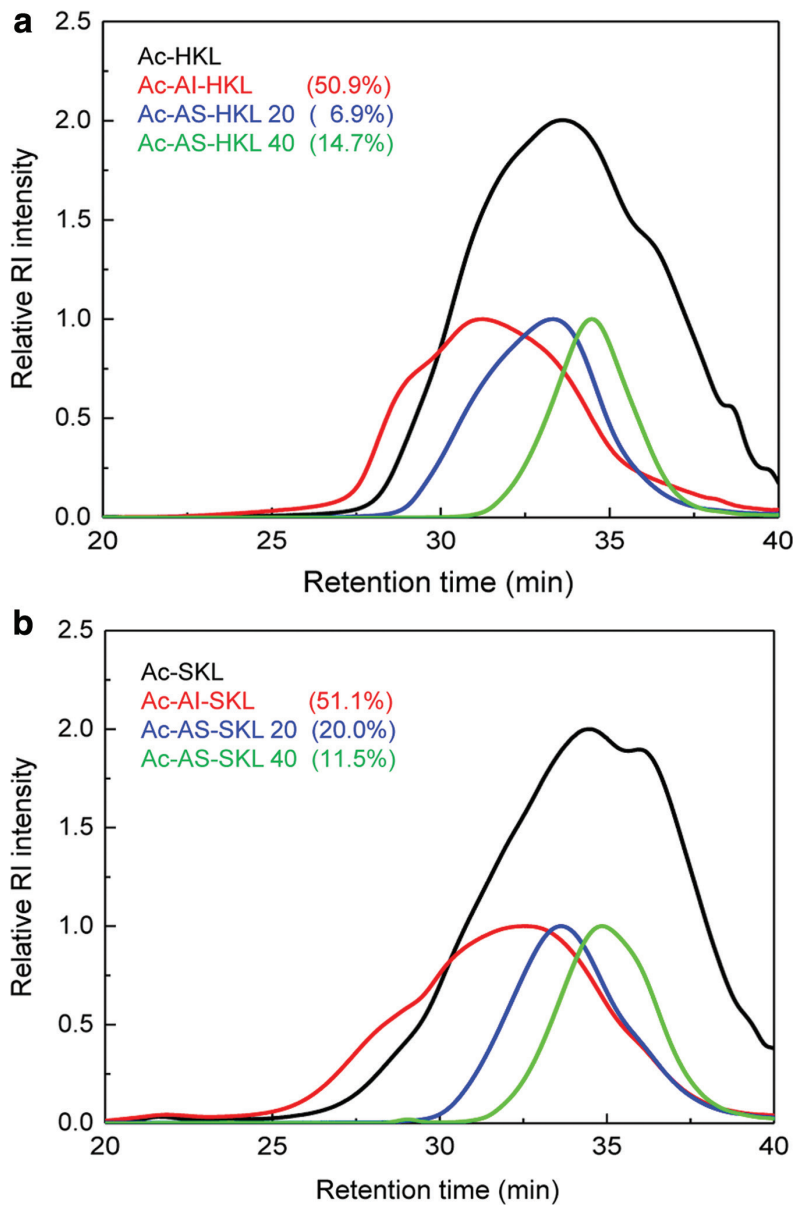

Figure 3: SEC-RI chromatograms of (a) Ac-HKL and its fractions and (b) Ac-SKL and its fractions. The Y axis shows the relative RI intensity.
Table 1: $5-5^{\prime}$ abundance of kraft lignins and their fractions.

\begin{tabular}{|c|c|c|c|c|}
\hline \multirow[b]{3}{*}{$M-804^{\prime}$} & 5-5' Type & G-type & S-type & \multirow{2}{*}{$\begin{array}{r}\text { 5-5' abundance } \\
\text { (per } 100 \mathrm{Ar} \text { ) }\end{array}$} \\
\hline & & \multicolumn{2}{|c|}{$\left(\mathrm{mmol} \mathrm{g}^{-1}\right)$} & \\
\hline & - & 0.519 & - & 0.0 \\
\hline \multicolumn{5}{|l|}{ Softwood KL } \\
\hline SKL & 0.071 & 0.262 & - & 17.4 \\
\hline Al-SKL & 0.047 & 0.141 & - & 20.0 \\
\hline AS-SKL20 & 0.100 & 0.457 & - & 15.2 \\
\hline AS-SKL40 & 0.069 & 0.288 & - & 16.2 \\
\hline \multicolumn{5}{|l|}{ Hardwood KL } \\
\hline HKL & 0.027 & 0.142 & 0.209 & 6.7 \\
\hline $\mathrm{Al}-\mathrm{HKL}$ & 0.032 & 0.062 & 0.132 & 12.3 \\
\hline AS-HKL20 & 0.041 & 0.160 & 0.259 & 8.2 \\
\hline AS-HKL40 & 0.019 & 0.085 & 0.147 & 7.1 \\
\hline
\end{tabular}

abundances were enriched in AI-SKL and AI-HKL from untreated KLs (20.0 and 12.3 per 100 Ar, respectively). With respect to the precipitates in the acetone-hexane system, no significant difference between AS-SKL20 and AS-SKL40 was observed (15.2 and 16.2 per $100 \mathrm{Ar}$, respectively) regardless of their different MM distributions; this tendency was also applicable to AS-HKL20 and AS-HKL40 (8.2 and 7.1 per 100 Ar, respectively). Crestini et al. (Crestini et al. 2017) determined that the AI-KL fraction retains a higher content of all native lignin interunit linkage, while AS-KLs, smaller MM fractions, were the products severely degraded by kraft cooking. Dramatic structural changes in AS-KLs were corroborated by their decreased polydispersity indexes, increased phenolic hydroxyl group, a decrease in aromatic $\mathrm{C}-\mathrm{H}$, and the loss of aliphatic sidechain. The lowered 5-5' abundances of AS-KLs in this study can also be explained by the latter two rationales.

Then, the relationship between $\mathrm{MM}$ and 5-5' abundances were validated. As shown in Figure 3, the peak top retention times of Ac-AI-KL, Ac-AS-KL20 and Ac-AI-KL40 were approximately 31, 33 and $35 \mathrm{~min}$, respectively. The MM values at these retention times were plotted vs. the 5-5' abundance, and linear fittings with determination coefficients $\left(\mathrm{R}^{2}\right)$ are shown in Figure 4a. The " $\mathrm{r}$ " values in the figure represent hydrodynamic radii at corresponding retention times. The linear fittings of 33 and 31 min showed moderate to good positive correlations with the $\mathrm{R}^{2}$ values of 0.547 and 0.737 , respectively. Meanwhile, there was no correlation with that of $35 \mathrm{~min}\left(R^{2}=0.039\right)$. Because MALS has higher accuracy for larger molecules, the correlation of AIKL fractions at 25 min was also examined, as shown in Figure 4b. Interestingly, an excellent correlation with an $\mathrm{R}^{2}$ value of 0.991 was obtained for these fractions. The results demonstrated that MM 

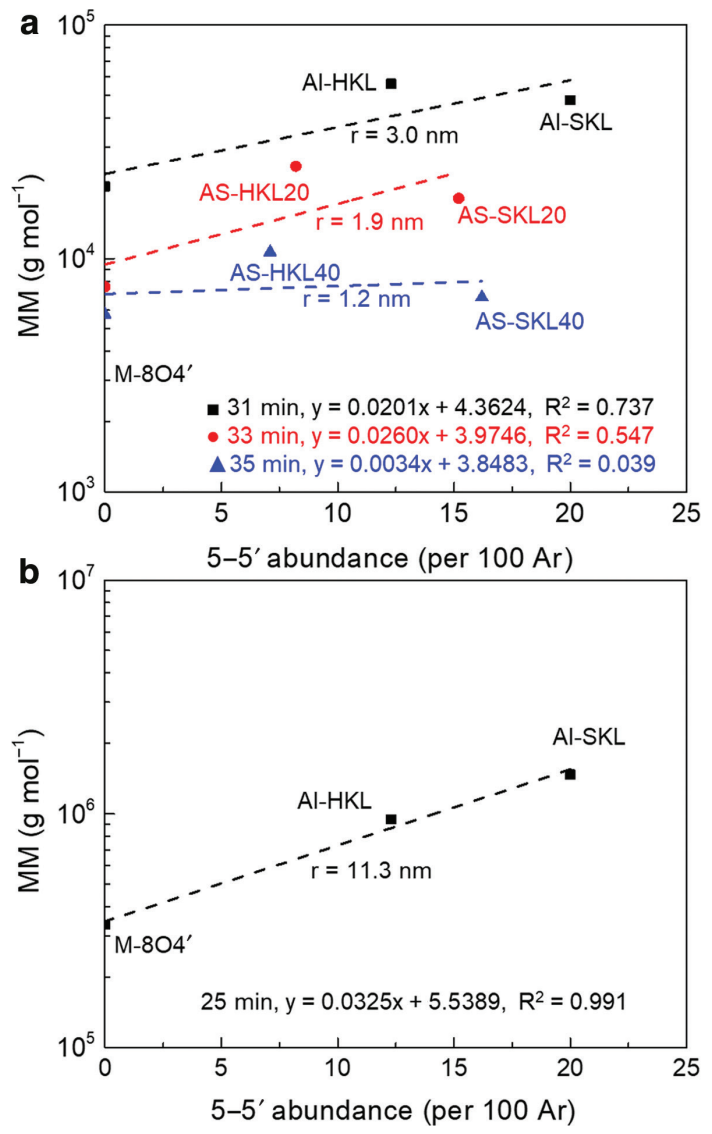

Figure 4: Molar mass of sample vs. the 5-5' abundance of lignin fractions (a) at different retention times and (b) at $25 \mathrm{~min}$. The "r" value shows the hydrodynamic radius of each retention time.

is proportional to the $5-5^{\prime}$ abundance of lignin samples, and the superior linearity is acquired for higher MM fractions. The poor correlation of the 35-min fractions may be ascribed to condensed structures formed during kraft cooking that are not present in native lignins (Crestini et al. 2017). These results show that branched substructures in lignin molecules result in less swollen, densely packed structure, and this trend is more valid for lignins with larger molecular sizes.

\section{Conclusion}

The difference in MM between the linear lignin model and KLs was clearly revealed using SEC-MALS equipped with a $785 \mathrm{~nm}$ laser light and bandpass filters. At the same retention time, the MMs of KLs were always larger than that of the linear lignin model; thus, KL has a more compact solution structure than those of the linear 8-0-4' model and polystyrene. Such a morphological gap can lead to the underestimation of lignin MM by the conventional relative MM measurement.

An easy method, nitrobenzene oxidation combined with ${ }^{1} \mathrm{H}$ NMR spectroscopy, was used to estimate the 5-5' interunit linkage of these three lignin samples and their fractions. The relationship between the $\mathrm{MM}$ and $5-5^{\prime}$ abundance was determined to be positively correlated, especially for the high MM molecules. It is clearly revealed that for lignins with the same large molecular size, the more branched structure results in a denser morphology.

The insight gained from this study provides a new platform for predicting mechanical and rheological properties, especially of technically important high MM lignins.

Author contributions: All the authors have accepted responsibility for the entire content of this submitted manuscript and approved submission.

Research funding: A part of this research was financially supported by JSPS KAKENHI [Grant-in-Aid for Scientific Research (A)], Funder Id: http://dx.doi. org/10.13039/501100001691, grant nos. 26252022 and $18 \mathrm{H} 03954$.

Employment or leadership: None declared. Honorarium: None declared.

\section{References}

Alekhina, M., Ershova, O., Ebert, A., Heikkinen, S., Sixta, H. (2015) Softwood kraft lignin for value-added applications: fractionation and structural characterization. Ind. Crop. Prod. 66:220-228.

Alliet, D.F., Pacco, J.M. (1968) The investigation of parallel-column systems in gel permeation chromatography. J. Polym. Sci. Pol. Sym. 21:199-213.

Baumberger, S., Abaecherli, A., Fasching, M., Gellerstedt, G., Gosselink, R., Hortling, B., Li, J., Saake, B., Dejong, E. (2007) Molar mass determination of lignins by size-exclusion chromatography: towards standardisation of the method. Holzforschung 61:459-468.

Bose, S.K., Wilson, K.L., Francis, R.C., Aoyama, M. (1998) Lignin analysis by permanganate oxidation. I. native spruce lignin. Holzforschung 52:297-303.

Capanema, E.A., Balakshin, M.Y., Chen, C., Gratzl, J.S., Gracz, H. (2001) Structural analysis of residual and technical lignins by $1 \mathrm{H}-13 \mathrm{C}$ correlation 2D NMR-spectroscopy. Holzforschung 55:302-308.

Capanema, E.A., Balakshin, M.Y., Kadla, J.F. (2004) A comprehensive approach for quantitative lignin characterization by NMR spectroscopy. J. Agric. Food. Chem. 52:1850-1860.

Chang, H., Jiang, X. (2020) Biphenyl structure and its impact on the macromolecular structure of lignin: a critical review. J. Wood. Chem. Technol. 40:81-90. 
Crestini, C., Melone, F., Sette, M., Saladino, R. (2011) Milled wood lignin: a linear oligomer. Biomacromolecules 12:3928-3935.

Crestini, C., Lange, H., Sette, M., Argyropoulos, D.S. (2017) On the structure of softwood kraft lignin. Green. Chem. 19:4104-4121.

Cui, C., Sun, R., Argyropoulos, D.S. (2014) Fractional precipitation of softwood kraft lignin: isolation of narrow fractions common to a variety of lignins. ACS. Sustain. Chem. Eng. 2:959-968.

Erickson, M., Larsson, S., Miksche, G.E. (1973) Gaschromatographische analyse von ligninoxydations-produkten. Viii.* Zur Struktur Des Lignins Der Fichte. Acta. Chem. Scand. 27:903-914.

Glasser, W.G., Dave, V., Frazier, C.E. (1993) Molecular weight distribution of (semi-) commercial lignin derivatives. J. Wood. Chem. Technol. 13:545-559.

Goring, D.A.I. (1971) Polymer properties of lignin and lignin derivatives. In: Lignins. Occurrence, Formation, Structure, and Reactions. Eds. Sarkanen, K.V., Ludwig, C.H. Wiley-Interscience, New York. pp. 695-768.

Gosselink, R., Abächerli, A., Semke, H., Malherbe, R., Käuper, P., Nadif, A., Van dam, J. (2004) Analytical protocols for characterisation of sulphur-free lignin. Ind. Crop. Prod. 19:271-281.

Katahira, R., Nakatsubo, F. (2001) Determination of nitrobenzene oxidation products by GC and $1 \mathrm{H}$-NMR spectroscopy using 5-iodovanillin as a new internal standard. J. Wood. Sci. 47:378-382.

Kishimoto, T., Uraki, Y., Ubukata, M. (2006) Chemical synthesis of $\beta$-0-4 type artificial lignin. Org. Biomol. Chem. 4:1343-1347.

Kishimoto, T., Uraki, Y., Ubukata, M. (2008a) Synthesis of $\beta$-0-4-type artificial lignin polymers and their analysis by NMR spectroscopy. Org. Biomol. Chem. 6:2982-2987.

Kishimoto, T., Uraki, Y., Ubukata, M. (2008b) Synthesis of bromoacetophenone derivatives as starting monomers for $\beta$-0-4 type artificial lignin polymers. J. Wood. Chem. Technol. 28:97-105.

Lindströmn, T. (1979) The colloidal behaviour of kraft lignin. Colloid. Polym. Sci. 257:277-285.

Lourençon, T.V., Hansel, F.A., Silva, T.A.D., Ramos, L.P., Muniz, G.I.B.D., Magalhães, W.L.E. (2015) Hardwood and softwood kraft lignins fractionation by simple sequential acid precipitation. Sep. Purif. Technol. 154:82-88.

Lue, L. (2000) Volumetric behavior of a thermal dendritic polymers: Monte Carlo simulation. Macromolecules 33:2266-2272.

Lyulin, A.V., Adolf, D.B., Davies, G.R. (2001) Computer simulations of hyperbranched polymers in shear flows. Macromolecules 34:3783-3789.
Pew, J.C. (1963) Evidence of a biphenyl group in lignin1. J. Org. Chem. 28:1048-1054.

Ralph, S.A., Ralph, J., Landucci, L., Landucci, L. (2004) NMR database of lignin and cell wall model compounds. (http://www. glbrc.org/database_and_software/NMRdatabase).

Saito, T., Perkins, J.H., Vautard, F., Meyer, H.M., Messman, J.M., Tolnai, B., Naskar, A.K. (2014) Methanol fractionation of softwood kraft lignin: impact on the lignin properties. ChemSusChem 7:221-228.

Santos, R.B., Capanema, E.A., Balakshin, M.Y., Chang, H., Jameel, H. (2011) Effect of hardwoods characteristics on kraft pulping process: emphasis on lignin structure. Bioresources 6:3623-3637.

Siochi, E.J., Ward, T.C., Haney, M.A., Mahn, B. (1990) The absolute molecular weight distribution of hydroxypropylated lignins. Macromolecules 23:1420-1429.

Smit, R., Suckling, I., Ede, R. (1997) A new method for the quantification of condensed and uncondensed softwood lignin structures. Proceedings of $9^{\text {th }}$ International Symposium on Wood and Pulping Chemistry, Montreal, Canada.

Spatorico, A., Coulter, B. (1973) Molecular weight determinations by gel-permeation chromatography and viscometry. J. Polym. Sci. Pol. Phys. 11:1139-1150.

Tamai, A., Goto, H., Akiyama, T., Matsumoto, Y. (2015) Revisiting alkaline nitrobenzene oxidation: quantitative evaluation of biphenyl structures in cedar wood lignin (cryptomeria japonica) by a modified nitrobenzene oxidation method. Holzforschung 69:951-958.

Tarabanko, V., Tarabanko, N. (2017) Catalytic oxidation of lignins into the aromatic aldehydes: general process trends and development prospects. Int. J. Mol. Sci. 18:2421.

Villar, J., Caperos, A., García-Ochoa, F. (1997) Oxidation of hardwood kraft-lignin to phenolic derivatives. nitrobenzene and copper oxide as oxidants. J. Wood. Chem. Technol. 17: 259-285.

Wang, L., Uraki, Y., Koda, K., Gele, A., Zhou, X., Chen, F. (2019) Determination of the absolute molar mass of acetylated eucalyptus kraft lignin by two types of size-exclusion chromatography combined with multi-angle laser light-scattering detectors. Holzforschung 73:363-369.

Zinovyev, G., Sulaeva, I., Podzimek, S., Rössner, D., Kilpeläinen, I., Sumerskii, I., Rosenau, T., Potthast, A. (2018) Getting closer to absolute molar masses of technical lignins. ChemSusChem 11:3259-3268. 